\title{
A microprocessor control and recording system for instrumental behavior research
}

\author{
WOLFGANG LEBER \\ Max-Planck-Institut für Hirnforschung, Neurobiologische Abteilung, Frankfurt a/M, West Germany
}

\begin{abstract}
This paper describes a miroprocessor system developed for experimental control and data collection in real time. By connecting the experimental equipment to the microprocessor via a serial communication interface, transmission of various animal test-chamber functions over greater distances with only a few cables is facilitated. The microprocessor is programmed on a host computer (PDP/8). A combined terminal station with a cassette deck is used for data and program storage.
\end{abstract}

This paper describes a microprocessor system developed for laboratory research in instrumental learning. Animal subjects are used in experiments in which reinforcement schedules may be varied and lever responses required, or reaction times measured in a jumping platform situation. The animals are run in individual test chambers (boxes). Research in our laboratory is concerned with behavioral changes caused by pharmacological, electrical, or chemical manipulation of various central nervous subsystems. The ability to quickly change an experimental control program is crucial. Microprocessors offer a number of advantages for fulfilling our needs.

\section{SYSTEM DESCRIPTION}

The choice of a microprocessor system was based on the program aids at our disposal. A three-pass assembler (similar to PAL III) for the Digital Equipment PDP. 8 was available to program the 8000 family (8008, 8080,8085, Z80). We have several PDP-8s with convenient peripheral equipment (disk storage, LP cassette tape units, display terminals) at our disposal that are excellent as host computers.

Cross-assemblers are now available for all established microprocessors, such as $8080,8085, \mathrm{Z80}, 6800,6502$, and F8, and are included in the manufacturer's sales lists. For owners of a minicomputer from the PDP-8 family, the IM 6100 microprocessor is also of interest. It is compatible with PDP-8 software, thus eliminating the necessity of a cross-assembler for host programming.

The interaction of the hardware elements of the experimental control system is illustrated in Figure 1.

\section{The Central Processing Unit}

This module contains a central processing unit (CPU) of the 8000 family, a $4 \mathrm{~K}$ random-access memory (RAM), an asynchronous interface (current loop 110-2,400 baud), and an address decoder for generating IOT commands. Due to the module's high integration density, it was not possible to connect a console directly to the CPU; thus the control panel had to be installed as a peripheral unit with special features. A minimal operation program is necessary that, together with the bootstrap, is easily stored in a read-only memory (ROM).

Especially during the process of developing the program, one should be able to interrupt the program flow over the control panel; for example, when checking out on-line, loading, or partial program starts. Interrupts can be recognized by an additional interface, so that the respective vector start address can also be produced to handle the interrupt. In our experiments, for example, interrupts were caused by animal responses, impulses from the real-time clock, and from the arithmetic unit (APU).

\section{The Real-Time Clock}

A crystal-controlled real-time clock produces program interrupts at 1-sec intervals, thus making a fine graduation of program flow possible; for example, for the marking of experimental steps. A $10-\mathrm{msec}$ cycle activates a counter for the measurement of reaction time. To relieve the CPU, we chose an external decimal counter with four decades that can be queried in parallel by the program as necessary.

\section{Random-Number Generator}

A generator was developed that produces equally distributed random impulses as well as preprogrammable normal or binomial distributions (Massen \& Kohl, 1975). Depending on the program condition, interrupts can be released by this "noise generator" or the presence of an impulse from the noise generator can be tested.

\section{Arithmetic Unit}

To relieve the CPU, we used an APU for arithmetic problems. The APU is considered the computing "slave" of the central unit; that is, instructions to process numerical values are delegated by the program to the APU. While the CPU carries out control functions, the APU only reports back when the arithmetic operations are completed. 


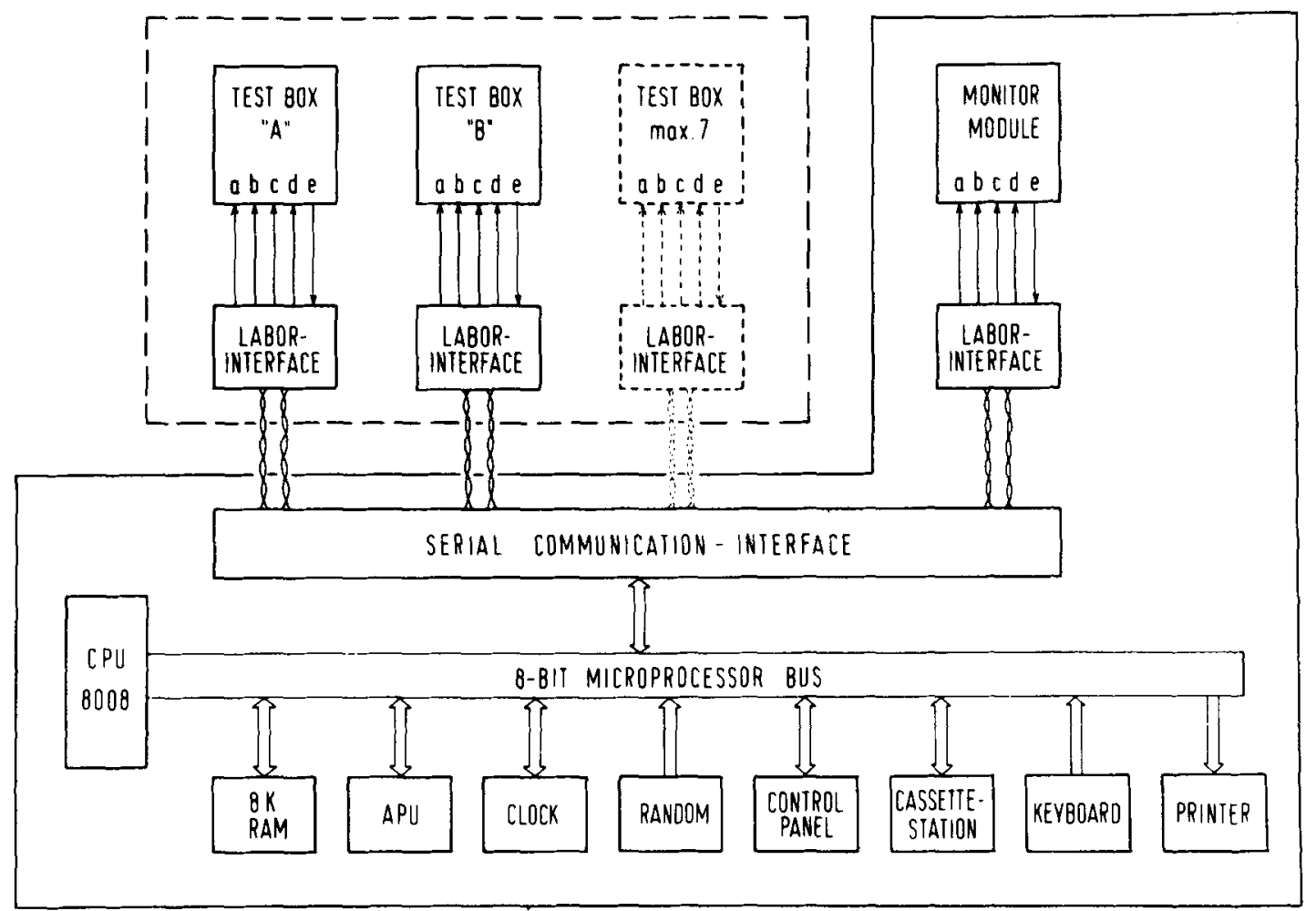

Figure 1. Block diagram of the microprocessor control and data acquisition system. The control panel, the real-time clock, the random ascent generator, the arithmetic unit, the terminal with keyboard, the printer and the double cassette drive are connected over the MPS-BUS, 28 well 28 the communications interface, which is connected by twisted pair to the modules. These modules are located on the Skinner box.

\section{The Serial Communications Interface}

The distance between the control system and the animal test chambers makes the use of fewer and cheaper connecting cables desirable. Serial data transfer offers a feasible alternative to the extensive wiring usually required. Inexpensive twisted-pair wires enable a largely errorless transfer of up to about 1 Mbaud.

The interface attached to the $1 / 0$ port on the microprocessor bus is divided into three functional blocks: (1) parallel-serial converter with line driver for the transmission of a stimulus pattern; (2) parallelserial converter with line driver for transmission of various releasing functions (e.g., the release of food pellets into the experimental chamber); (3) input port with an event detector to generate an interrupt so that activities from the test boxes can be processed.

Due to its relatively long word length (26 bits), the parallel-serial converter is built with a conventional parallel-in/serial-out shift register (74165). If word lengths of 8 bits per transmission block are sufficient,

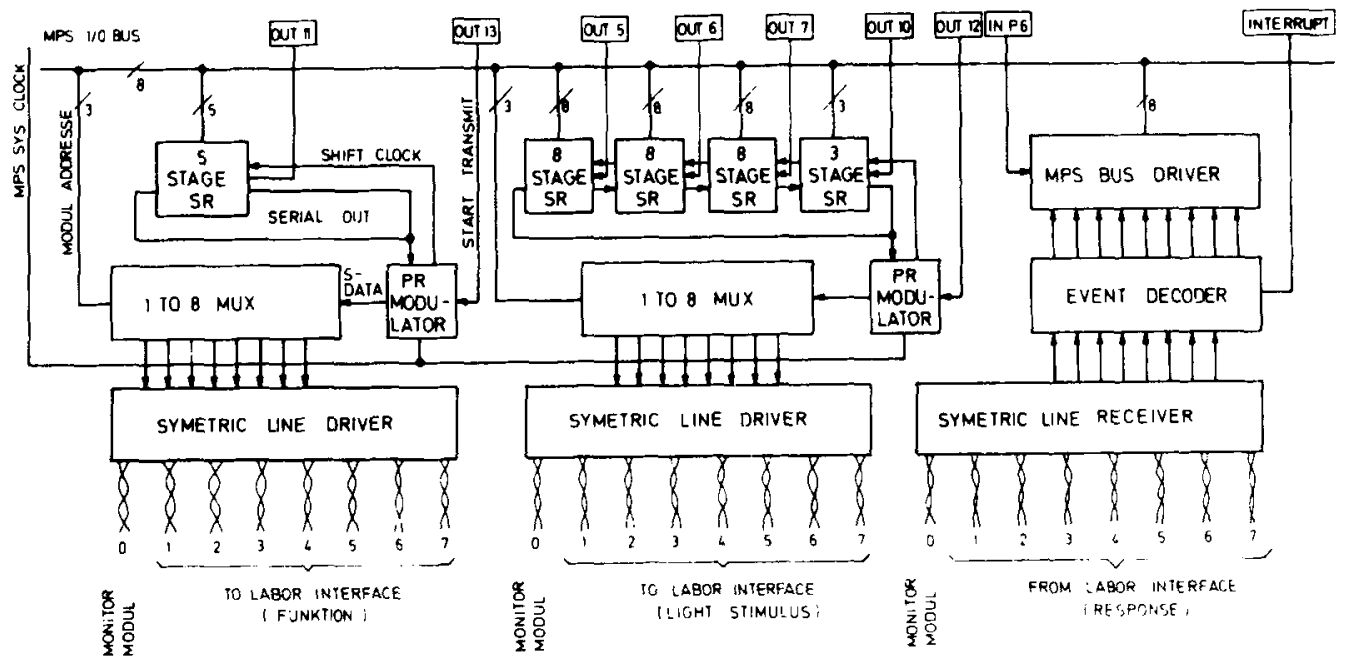

Figure 2. Block diagram of the serial communication interface. 


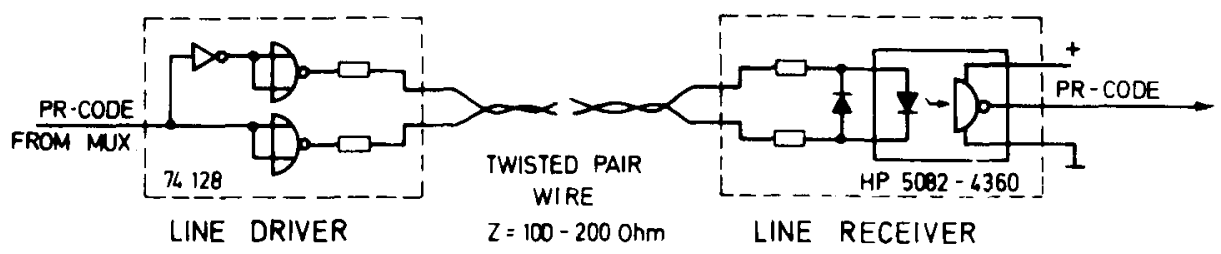

Figure 3. Example of the circuit of a symmetric data transmission unit.

then "UARTs" can be used more advantageously. A complete stimulus pattern is composed of 25 bit points and 1 bit for color information. An output sequence of 4 bytes is therefore necessary to load the shift register. A further output command initiates transfer to the laboratory interface, which is addressed with the data on the bus at that time ( 3 bits is equivalent to eight lab interfaces). A second independent 5-bit shift register transfers "functions"; for example, to turn on the shock in the animal test chamber. Both kinds of shift registers are designed to recirculate the data. The most recently loaded data can be transferred to various laboratory interfaces in a very short time; for example, when the same pattern is to be presented in all the test chambers at once.

The data are transferred in pulse ratio (PR) code via a line driver (74128) that is adapted to a line impedance of about $Z=100-200 \mathrm{ohm}$. The speed of data transfer is derived from the microprocessor system clock. We dispensed with a serial transfer of information from the test chambers because we process only one type of response per chamber. A symmetric line with an optically coupled isolator as a line receiver for galvanic isolation is also used here for interference-free transfer. The entire input information is available simultaneously

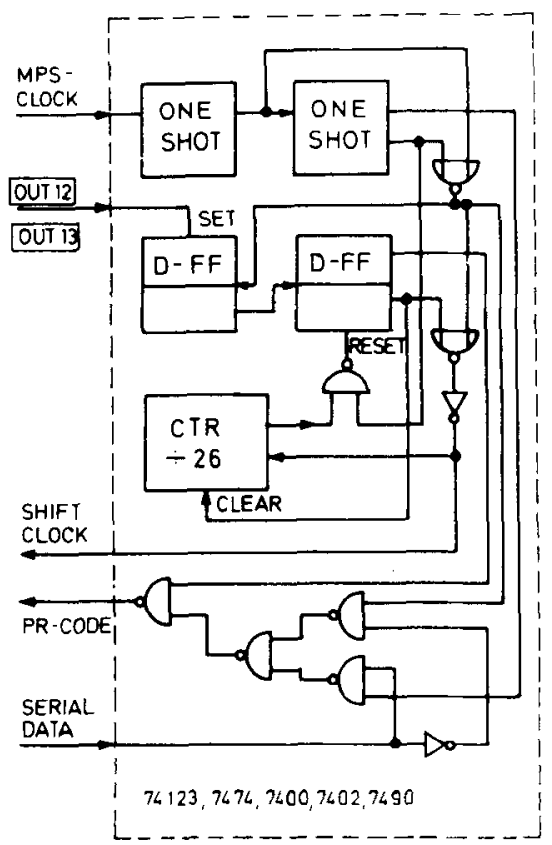

Figure 4. Circuit of the pulse ratio modulator. in 1 byte on the input port. A change in any one of the inputs produces an interrupt with top priority in the microprocessor.

\section{Laboratory Interface}

Each of the laboratory interfaces is connected by three twisted-pair wires to the control equipment. As a rule, several test chambers are installed in one room so that operating power can be obtained from a simple power supply unit. The operating power is regulated in each interface separately.

The basic logic is functionally complementary to that of the communications interface, for example, serial-toparallel conversion. The circuit for symmetrical data transmission is shown in Figure 3. The shift clock is easily separated from the data signal by means of a one shot. Another one shot is adapted to the length of the data record and generates a transfer pulse to store the data in an output buffer register (see Figure 6). An interface driver (75752) is attached so that lamps or relays up to $30 \mathrm{~V}$ and $300 \mathrm{~mA}$ can be used. The "functions" part controls four relays. These contacts

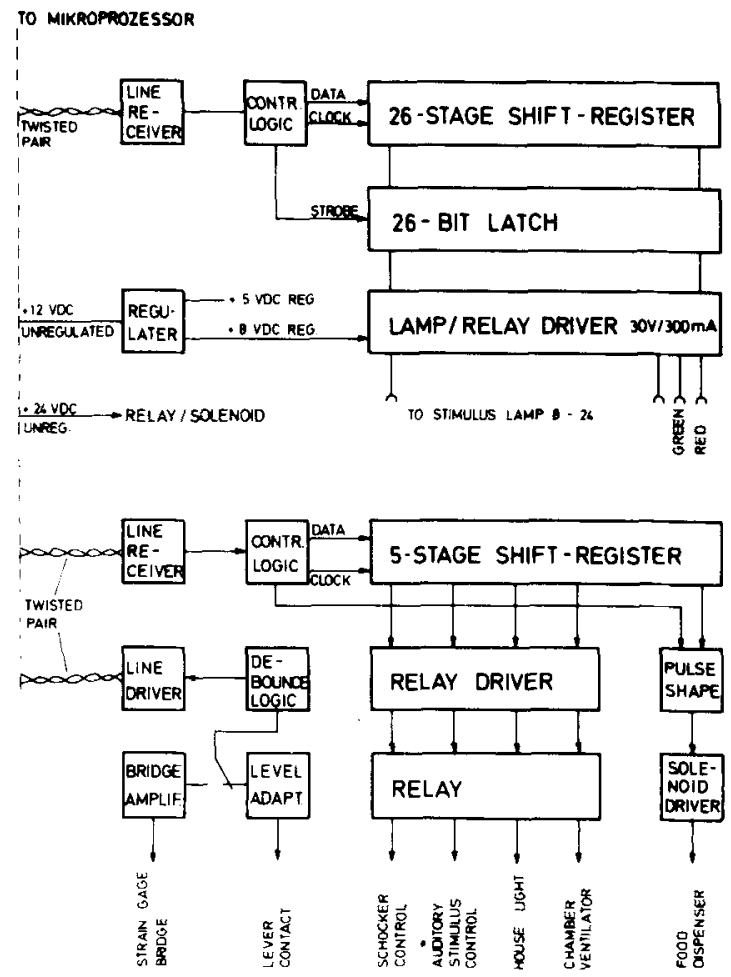

Figure 5. Block diagram of the laboratory interface. 


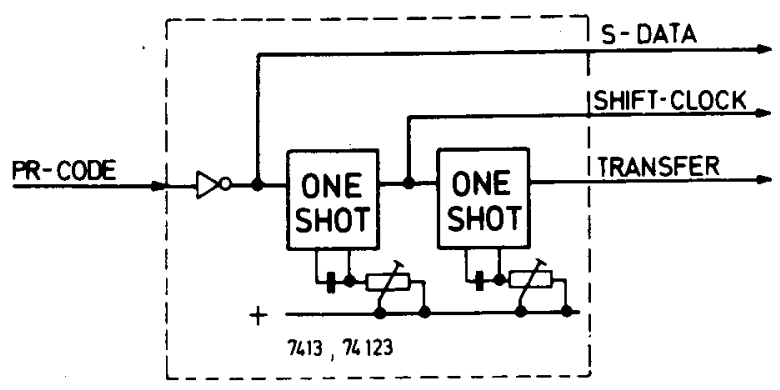

Figure 6. Circuit of the pulse ratio demodulators.

(NO/NC) can be used to fulfill various needs; for example, to release shock or to control a ventilator. Operation of the food pellet dispenser is an exception. Here, step motors or solenoids, which have to be activated for a defined period $(40 \mathrm{msec}, 1 \mathrm{sec}$, etc.), are also employed. A one shot, with timing programmed by means of the resistance in the step motor or solenoid plug, relieves the microprocessor of this tedious job.

Experimental subject responses can be registered in two different ways: (1) Leverpresses-A NO/NC contact is debounced with a set-reset FF. (2) Jumping platform responses-We chose a bridge circuit with a strain gauge to generate a contactless occupied signal. An op-amp proved to be sufficient to generate on-off signals (Figure 7). This circuit gives satisfactory results for animal weights from a minimum of $60 \mathrm{~g}$ on an aluminum plate $150 \times 100 \times 2.5 \mathrm{~mm}$. Separate plugs connect the various elements on the text box with the interface. This enables a quick change of parts when necessary.

\section{Monitor Module}

The monitor module, which contains a laboratory interface, assists in checking the program and in controlling the experimental situation. All modules connected to the experimental control system can be simulated by a selection dial on the monitor module; for example, to verify that food pellets were released when a lever was pressed.

\section{Input-Output Devices}

A Terminal Silent 733 (Texas Instruments) is connected over a serial 1,200-baud current loop interface for data input and output. In addition to a keyboard and a thermoprinter, this terminal also contains two cassette tape units, one of which is used for program input, the other for storage of experimental data. A remote device control option allows for complete control of the terminal from the microprocessor program.

\section{PROGRAM DEVELOPMENT}

All previously used programs were developed with a microprocessor language assembler (MLA) on a host computer (PDP-8). With the exception of simulation, all program development can take place on the minicomputer, since the MLA is implemented for the operating system OS-8.

We divided the program into software modules (i.e., into singular, frequently used subroutines) so programmed that they can be used for different control programs. These service routines are called up from central storage and loaded in the editor during program development as necessary.

The following examples of program modules illustrate the idea. (1) Input routines over the terminal keyboard; for example, animal number, experiment number, experimental condition, date, stimuli, and so on (see Figure 8). (2) Documentation routines: (a) storage of real-time data in the data carrier; (b) printout of events on the printer. (3) Functional routines depending on the reinforcement schedule: (a) presentation of stimuli; (b) administration of rewards (e.g., food); (c) time schedules according to the schedule of reinforcement. (4) Overall short evaluation of the results after each

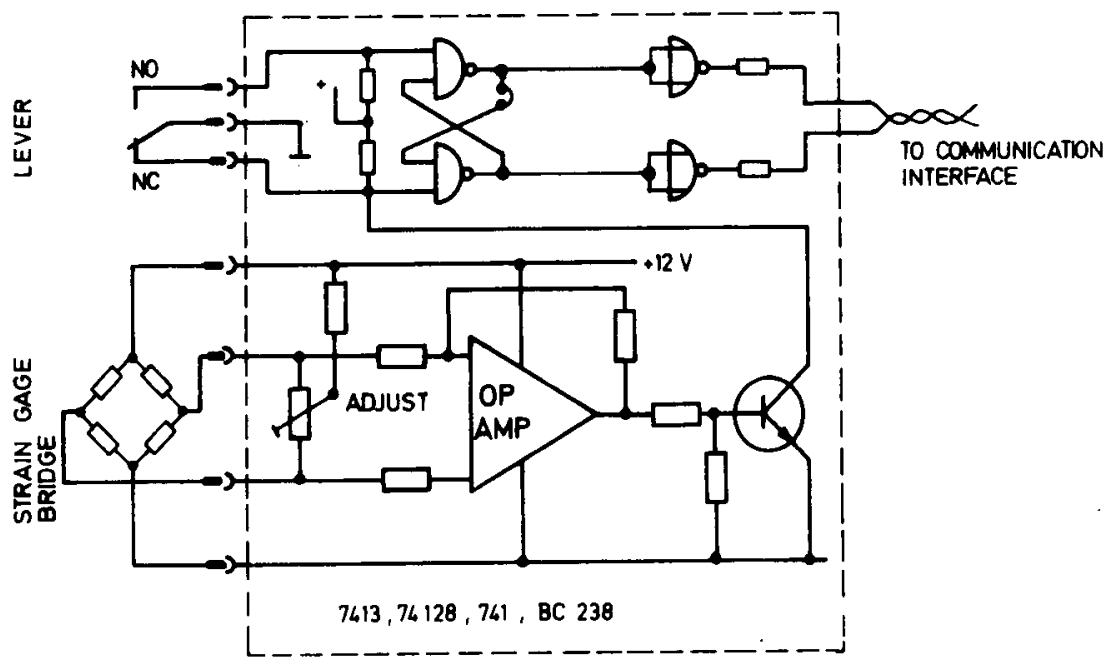

Figure 7. Circuit of the attachment of a lever or platform jumping stand. 


\begin{tabular}{|c|c|c|c|c|c|c|c|c|c|c|c|}
\hline \multicolumn{12}{|c|}{ VERSUCHSART :L } \\
\hline \multirow{2}{*}{\multicolumn{2}{|c|}{$\begin{array}{l}\text { VERSUCH NR } \\
\text { STIMULI BEZ }\end{array}$}} & :014 & & & & & & & & & \\
\hline \multirow{2}{*}{\multicolumn{12}{|c|}{ VERSUCHSTIER :18 }} \\
\hline & & & & & & & & & & & \\
\hline \multicolumn{2}{|c|}{ TIERGRUPPE } & :S & & & & & & & & & \\
\hline \multicolumn{2}{|c|}{ TESTBOX } & :B & & & & & & & & & \\
\hline \multicolumn{2}{|c|}{ DATUM } & $: 170377$ & & & & & & & & & \\
\hline \multicolumn{2}{|c|}{ BEGINN } & 1044 & & & & & & & & & \\
\hline \multicolumn{12}{|c|}{ DAUER (UNITS) :07 } \\
\hline \multicolumn{12}{|c|}{ L014DE18S1703771044 } \\
\hline 0003 & $1 \mathrm{~B} 030 \bullet$ & 0043 & $1 \mathrm{~B} 384$ & 0072 & $1 \mathrm{~B} 270$ & 0111 & $1 \mathrm{~B} 179$ & 0147 & $1 \mathrm{~B} 732$ & 0185 & $1 \mathrm{~B} 560$ \\
\hline 0024 & $1 \mathrm{~B} 401 \bullet$ & 0274 & $1 \mathrm{~B} 431$ • & 0401 & $1 \mathrm{~B} 149$ & 0437 & $1 \mathrm{~B} 746$ & 0476 & $1 \mathrm{~B} 684$ & 0513 & $1 B 324$ \\
\hline 0517 & $1 \mathrm{~B} 400$ & 0570 & $1 \mathrm{~B} 074$ & 0636 & $1 \mathrm{~B} 646 \bullet$ & 0687 & $1 \mathrm{~B} 778$ & 0725 & $1 \mathrm{~B} 533$ & 0763 & 1B334. \\
\hline 0801 & $1 B 119$. & 0854 & $1 \mathrm{~B} 446$ & 0892 & $1 \mathrm{~B} 257$ & 0921 & $1 B 150$ & 0927 & $1 \mathrm{~B} 597$ & 0943 & $1 \mathrm{~B} 425$ \\
\hline 0937 & $1 \mathrm{~B} 350$ & 0952 & $1 \mathrm{~B} 268$ & 0994 & $1 \mathrm{~B} 444$ & 1024 & $1 \mathrm{~B} 402$ & 1062 & $18231 *$ & 1100 & 1 B098. \\
\hline 1147 & $1 \mathrm{~B} 787$. & 1157 & $1 B 729$ & 1207 & $1 \mathrm{~B} 711 \bullet$ & 1244 & $1 \mathrm{~B} 426$ & 1284 & $1 \mathrm{~B} 442$ & 1332 & $1 \mathrm{~B} 207$ 。 \\
\hline 1371 & $1 \mathrm{~B} 124 \bullet$ & 1413 & 1B379• & 1454 & $1 \mathrm{~B} 480$ & 1492 & $1 \mathrm{~B} 254 \bullet$ & 1530 & $1 \mathrm{~B} 060$ & 1566 & $1 \mathrm{~B} 636$ \\
\hline 1596 & $1 \mathrm{~B} 638$ & 1601 & $1 \mathrm{~B} 119$ & 1633 & 1B321 & 1646 & $1 \mathrm{~B} 698$ & 1652 & $1 \mathrm{~B} 262$ & 1691 & 1B104. \\
\hline 1727 & $1 \mathrm{~B} 778$ & 1765 & $1 \mathrm{~B} 583$ & 1813 & $1 \mathrm{~B} 374$ & 1851 & $1 \mathrm{~B} 133$ & 1882 & $1 \mathrm{~B} 232$ & 1920 & $1 \mathrm{~B} 054$ \\
\hline 1950 & $1 \mathrm{~B} 068$ & 1986 & $1 \mathrm{~B} 6533^{\circ}$ & 1993 & $1 \mathrm{~B} 389$ & 2033 & $1 \mathrm{~B} 325$ & 2086 & $1 \mathrm{~B} 646$ & 2146 & $1 \mathrm{~B} 692$ \\
\hline \multirow{2}{*}{\multicolumn{2}{|c|}{ SEGMENTE }} & \multirow{2}{*}{\multicolumn{10}{|c|}{$: 0 \times 256+224$}} \\
\hline & & & & & & & & & & & \\
\hline \multicolumn{2}{|c|}{ HDR TOTAL } & \multirow{2}{*}{\multicolumn{2}{|c|}{$: 0 \times 256+061$}} & & & & & & & & \\
\hline \multicolumn{2}{|c|}{ HDR POSITIV } & & & & & & & & & & \\
\hline
\end{tabular}

Figure 8. Protocol of a DRL experiment.

experiment (Figure 8). (5) Further routines: (a) interrupt processing (leverpressing, real-time clock, current outage, etc.); (b) numeric transformation routines (octal to decimal, etc.).

\section{PROGRAM STRUCTURE}

A detailed description of the program is quite involved and, for most readers, too specialized. Here, the basic idea is illustrated by selected program parts. (Detailed information may be obtained by writing the author.)

A program run is a repetition of sequences that consist of eight 1-sec steps each. During Step 3 or Step 4, depending on the schedule, a stimulus can be presented. With the start of Step 0, a documentation of the preceding sequence is released, provided that a random stimulus was presented and no reaction occurred during this time. In this way, concise documentation of the experimental run is assured.

One basic software module is "clock." The realtime clock generates an interrupt every second. In turn, this initiates processing of the clock routine, basically covering the division into segments, stimulus presentation (depending on the randomized routine), and measurement of total experiment time. The input instruction "INP4" resets the interrupt request from the clock. Restoring the old register contents completes this interrupt service routine.

If two different light patterns serve as stimuli, which of the two sequential patterns is presented during Step 3 or 4 is determined at the experimental parameter input. A stimulus pattern consists of 4 bytes. The output port is loaded sequentially according to the following pattern:

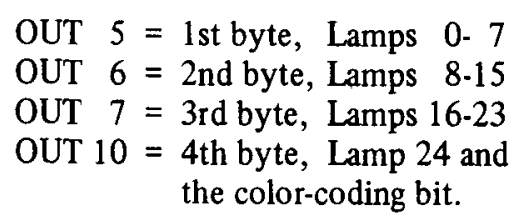

Transfer to the laboratory interface is initiated by the output instruction "OUT 12."

An answer routine from a fixed-interval program was chosen as a further example. Since the animals' activities can periodically exceed the maximum printout speed possible with 1,200 baud, a data stack is used for temporary storage. With the instruction "OUT 11," the data are loaded into the function register, and with "OUT 13," they are transferred to the respective laboratory interface.

\section{Real-Time Documentation}

We place special emphasis on perfect documentation of all data pertaining to the experiment. To this end, a form with four functionally diverse documentation blocks is used. By a documentation block, we mean a complete set of data stored on tape for later analysis. For several reasons, it seemed better to document in ASCII code rather than in packed binary data. In this way, every data cassette can also be inspected off-line at the terminal. There is no confusion with the control codes of the remote device controller and, parallel to data input, a protocol can be printed out on the thermoprinter. For block identification and termination, the control characters DC 1.4 and EOM are used, respectively.

\section{Parameter Block}

This block contains the information shown in 
Table 1

\begin{tabular}{ll}
\hline \multicolumn{1}{c}{ Questions } & \multicolumn{1}{c}{ Possible Answers } \\
\hline Type of Experiment: & A-Z \\
Experiment Number: & $000-999$ \\
Stimulus Type: & $\mathrm{A}-\mathrm{Z} ; \mathrm{A}-\mathrm{Z}$ \\
Animal Number: & $00-99$ \\
Test Box Number: & $\mathrm{A}-\mathrm{Z}$ \\
Date: & Day, month, year \\
Start: & Time (hours, minutes) \\
Length (Units): & Length of experiment \\
& (1 unit $=7$ min) \\
\hline
\end{tabular}

Table 1, which can be entered by the experimenter in the form of questions and answers at the beginning of the experiment.

As shown in Figure 8, the answer information in the first block (without questions) can be documented in summary on a cartridge. This is performed at the start of the experiment.

\section{Stimulus Block}

As opposed to the response block, only stimulus information not followed by a response (within the same experimental step) is documented in the stimulus block. The stimulus block contains information regarding the experimental step (EL), the stimulus (STI), the status (STA), and the text box (B).

\section{Response Block}

In addition to the elements of the stimulus block, information about the reaction time "RT" and the in terresponse time "IRT" (three-digit, in 10-msec intervals) as well as marking $\left(^{*}\right)$ for given rewards is included here.

\section{Comment Block}

The experimenter can document short commentaries over the keyboard during the experiment. This is useful for marking time-outs; for example, when the animal is asleep or engaged in nonexperimental activities.

\section{Preliminary Data Analysis}

To enable the experimenter to form an impression of the quantitative and qualitative performance of the animals after the experiment, a preliminary data analysis is printed out after each trial.

\section{EXAMPLE: ENVIRONMENTAL INFLUENCES ON BRAIN DEVELOPMENT IN TUPAIA GLIS (TREE SHREWS)}

The following example illustrates how our microprocessor system is used. In a series of experiments concerning environmental influences on brain development, tree shrews (Tupaia glis) were raised under various environmental conditions in order to study the effects on instrumental behavior in a Skinner box. The effects of various environmental manipulations were studied by using various reinforcement schedules: continuous reinforcement (CRF), fixed interval (FI), and differential reinforcement of low rates (DRL). Since the animals are usually at different learning stages in the experiment at any one time, easy program selection by quickly changing the cartridges between trials is a prerequisite for running the experiment smoothly.

Individual subject data were documented continuously in real-time on the cartridge, regardless of the various schedules. Real-time documentation enabled us to analyze variable constellations according to considerations that went beyond the experimental hypotheses. In later data analysis, differentiation of the animals according to the experimental parameters was easily accomplished. The printout provided a concrete overview of each animal's behavior.

In light of developments in microprocessor technology, a variety of advances in hardware and software are to be expected. In the past, programs were trimmed to use minimal storage space. This is now becoming less important because very inexpensive and highly integrated semiconductor memories are available.

\section{REFERENCE}

Massen, R., \& Kohl, A. Einfuhrung in die stochastische Rechentechnik. Electronik, 1975, 24, 55.

(Received for publication April 18, 1978; revision accepted July 6,1978 .) 